\title{
Progress on Dirac's Conjecture
}

\author{
Michael S. Payne* \\ Department of Mathematics and Statistics \\ The University of Melbourne \\ Melbourne, Australia \\ m. payne3@pgrad.unimelb .edu .au
}

\author{
David R. Wood ${ }^{\dagger}$ \\ School of Mathematical Sciences \\ Monash University \\ Melbourne, Australia \\ david.wood@monash.edu
}

Submitted: Sep 17, 2013; Accepted: Mar 27, 2014; Published: Apr 16, 2014

Mathematics Subject Classifications: 52C10, 52C30

\begin{abstract}
In 1951, Gabriel Dirac conjectured that every non-collinear set $P$ of $n$ points in the plane contains a point incident to at least $\frac{n}{2}-c$ of the lines determined by $P$, for some constant $c$. The following weakened conjecture was proved by Beck and by Szemerédi and Trotter: every non-collinear set $P$ of $n$ points in the plane contains a point in at least $\frac{n}{c^{\prime}}$ lines determined by $P$, for some constant $c^{\prime}$. We prove this result with $c^{\prime}=37$. We also give the best known constant for Beck's Theorem, proving that every set of $n$ points with at most $\ell$ collinear determines at least $\frac{1}{98} n(n-\ell)$ lines.
\end{abstract}

\section{Introduction}

Let $P$ be a finite set of points in the plane. A line that contains at least two points in $P$ is said to be determined by $P$. In 1951, Dirac [6] made the following conjecture, which remains unresolved:

Conjecture 1 (Dirac's Conjecture). Every non-collinear set $P$ of $n$ points in the plane contains a point in at least $\frac{n}{2}-c_{1}$ of the lines determined by $P$, for some constant $c_{1}$.

See reference [3] for examples showing that the $\frac{n}{2}$ bound would be tight. Note that if $P$ is non-collinear and contains $\frac{n}{2}$ or more collinear points, then Dirac's Conjecture holds. Thus we may assume that $P$ does not contain $\frac{n}{2}$ collinear points, and $n \geqslant 5$. In 1961, Erdős [7] proposed the following weakened conjecture.

Conjecture 2 (Weak Dirac Conjecture). Every non-collinear set $P$ of $n$ points contains a point in at least $\frac{n}{c_{2}}$ lines determined by $P$, for some constant $c_{2}$.

\footnotetext{
* Michael Payne is supported by an Australian Postgraduate Award from the Australian Government.
}

${ }^{\dagger}$ Research of David Wood is supported by the Australian Research Council. 
In 1983, the Weak Dirac Conjecture was proved independently by Beck [4] and Szemerédi and Trotter [19], in both cases with $c_{2}$ unspecified. In this paper we carefully combine and refine several known methods and results to give the best known value of $c_{2}$. See references $[8,9,11,13,17]$ for more on Dirac's Conjecture.

Theorem 3. Every non-collinear set $P$ of $n$ points contains a point in at least $\frac{n}{37}$ lines determined by $P$.

Theorem 3 is a consequence of the following theorem. The points of $P$ together with the lines determined by $P$ are called the arrangement of $P$.

Theorem 4. For every set $P$ of $n$ points in the plane with at most $\frac{n}{37}$ collinear points, the arrangement of $P$ has at least $\frac{n^{2}}{37}$ point-line incidences.

Proof of Theorem 3 assuming Theorem 4. Let $P$ be a set of $n$ non-collinear points in the plane. If $P$ contains at least $\frac{n}{37}$ collinear points, then every other point is in at least $\frac{n}{37}$ lines determined by $P$ (one through each of the collinear points). Otherwise, by Theorem 4 , the arrangement of $P$ has at least $\frac{n^{2}}{37}$ incidences, and so some point is incident with at least $\frac{n}{37}$ lines determined by $P$.

In his work on the Weak Dirac Conjecture, Beck proved the following theorem [4].

Theorem 5 (Beck's Theorem). Every set $P$ of $n$ points with at most $\ell$ collinear determines at least $c_{3} n(n-\ell)$ lines, for some constant $c_{3}$.

In Section 3 we use the proof of Theorem 4 and some simple lemmas to show that $c_{3} \geqslant \frac{1}{98}$. Similar methods and a bit more effort yield $c_{3} \geqslant \frac{1}{93}$ (see [16] for details).

\section{Proof of Theorem 4}

The proof of Theorem 4 takes inspiration from the well known proof of Beck's Theorem [5] as a corollary of the Szemerédi-Trotter Theorem [19], and also from the simple proof of the Szemerédi-Trotter Theorem due to Székely [18], which in turn is based on the Crossing Lemma. It is well known that Beck's proof can be used in conjunction with Székely's method to obtain a reasonably small constant for the Weak Dirac Conjecture. Reference [16] includes a self-contained exposition of these methods and a short proof with $c_{2}=2^{15}$.

The crossing number of a graph $G$, denoted by $\operatorname{cr}(G)$, is the minimum number of crossings in a drawing of $G$. The following lower bound on $\operatorname{cr}(G)$ was first proved by Ajtai et al. [2] and Leighton [12] (with worse constants). A simple proof with better constants than [2] and [12] can be found in [1]. The following version due to Pach et al. [15] is the strongest to date.

Theorem 6 (Crossing Lemma). For every graph $G$ with $n$ vertices and $m \geqslant \frac{103}{16} n$ edges,

$$
\operatorname{cr}(G) \geqslant \frac{1024 m^{3}}{31827 n^{2}}
$$




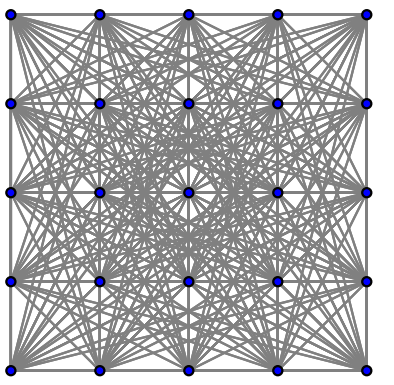

$G=G_{2}$

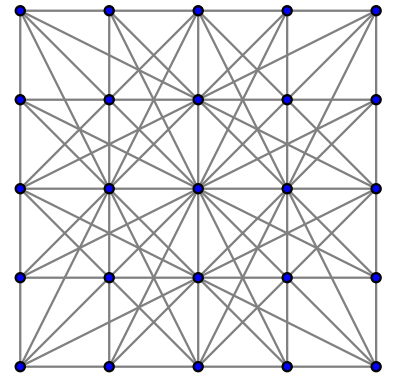

$G_{3}$

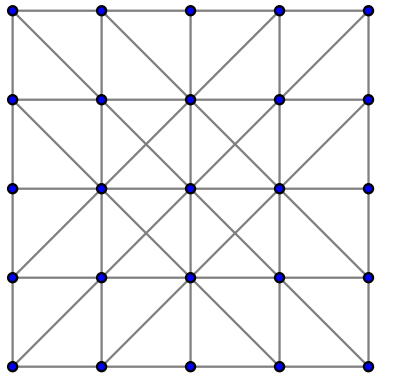

$G_{4}$

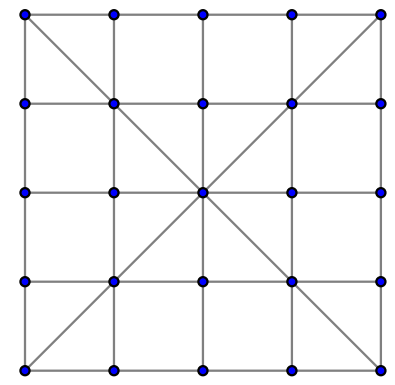

$G_{5}$

Figure 1: The graphs $G_{2}, G_{3}, G_{4}$, and $G_{5}$ in the case of the $5 \times 5$ grid.

In fact, we employ a slight strengthening of the Szemerédi-Trotter Theorem formulated in terms of visibility graphs. The visibility graph $G$ of a point set $P$ has vertex set $P$, where $v w \in E(G)$ whenever the line segment $v w$ contains no other point in $P$ (that is, $v$ and $w$ are consecutive on a line determined by $P$ ).

For $i \geqslant 2$, an $i$-line is a line containing exactly $i$ points in $P$. Let $s_{i}$ be the number of $i$-lines. Let $G_{i}$ be the spanning subgraph of the visibility graph of $P$ consisting of all edges in $j$-lines where $j \geqslant i$; see Figure 1 for an example. Note that since each $i$-line contributes $i-1$ edges, $\left|E\left(G_{i}\right)\right|=\sum_{j \geqslant i}(j-1) s_{j}$. Part (a) of the following version of the Szemerédi-Trotter Theorem gives a bound on $\left|E\left(G_{i}\right)\right|$, while part (b) is the well known version that bounds the number of $j$-lines for $j \geqslant i$.

Theorem 7 (Szemerédi-Trotter Theorem). Let $\alpha$ and $\beta$ be positive constants such that every graph $H$ with $n$ vertices and $m \geqslant \alpha n$ edges satisfies

$$
\operatorname{cr}(H) \geqslant \frac{m^{3}}{\beta n^{2}} \text {. }
$$

Let $P$ be a set of $n$ points in the plane. Then

$$
\begin{aligned}
\text { (a) } \sum_{j \geqslant i}(j-1) s_{j} & \leqslant \max \left\{\alpha n, \frac{\beta n^{2}}{2(i-1)^{2}}\right\}, \\
\text { and (b) } \sum_{j \geqslant i} s_{j} & \leqslant \max \left\{\frac{\alpha n}{i-1}, \frac{\beta n^{2}}{2(i-1)^{3}}\right\} .
\end{aligned}
$$

Proof. Suppose $\sum_{j \geqslant i}(j-1) s_{j}=\left|E\left(G_{i}\right)\right| \geqslant \alpha n$. Then by the assumed Crossing Lemma applied to $G_{i}$,

$$
\operatorname{cr}\left(G_{i}\right) \geqslant \frac{\left|E\left(G_{i}\right)\right|^{3}}{\beta n^{2}}=\frac{\left(\sum_{j \geqslant i}(j-1) s_{j}\right)^{2}\left|E\left(G_{i}\right)\right|}{\beta n^{2}} \geqslant \frac{(i-1)^{2}\left(\sum_{j \geqslant i} s_{j}\right)^{2}\left|E\left(G_{i}\right)\right|}{\beta n^{2}} .
$$

On the other hand, since two lines cross at most once,

$$
\operatorname{cr}\left(G_{i}\right) \leqslant\left(\begin{array}{c}
\sum_{j \geqslant i} s_{j} \\
2
\end{array}\right) \leqslant \frac{1}{2}\left(\sum_{j \geqslant i} s_{j}\right)^{2}
$$

Combining these inequalities yields part (a). Part (b) follows directly from part (a). 
The proof of Theorem 4 also employs Hirzebruch's Inequality [10].

Theorem 8 (Hirzebruch's Inequality). Let $P$ be a set of $n$ points with at most $n-3$ collinear. Then

$$
s_{2}+\frac{3}{4} s_{3} \geqslant n+\sum_{i \geqslant 5}(2 i-9) s_{i} .
$$

Theorem 9. Let $\alpha$ and $\beta$ be positive constants such that every graph $H$ with $n$ vertices and $m \geqslant \alpha$ edges satisfies

$$
\operatorname{cr}(H) \geqslant \frac{m^{3}}{\beta n^{2}}
$$

Fix an integer $c \geqslant 8$ and a real $\epsilon \in\left(0, \frac{1}{\alpha}\right)$. Let $h:=\frac{c(c-2)}{5 c-18}$. Then for every set $P$ of $n$ points in the plane with at most $\epsilon n$ collinear points, the arrangement of $P$ has at least $\delta n^{2}$ point-line incidences, where

$$
\delta=\frac{1}{h+1}\left(1-\epsilon \alpha-\frac{\beta}{2}\left(\frac{(c-h-2)(c+1)}{c^{3}}+\sum_{i \geqslant c} \frac{i+1}{i^{3}}\right)\right) .
$$

Theorem 4 follows from Theorems 6 and 9 by setting $\alpha=\frac{103}{16}, \beta=\frac{31827}{1024}, c=71$, and $\delta=\epsilon$, in which case $\delta \geqslant \frac{1}{36.158}$. The value of $\delta$ is readily calculated numerically since

$$
\sum_{i \geqslant c} \frac{i+1}{i^{3}}=\sum_{i \geqslant 1} \frac{i+1}{i^{3}}-\sum_{i=1}^{c-1} \frac{i+1}{i^{3}}=\zeta(2)+\zeta(3)-\sum_{i=1}^{c-1} \frac{i+1}{i^{3}}=2.847 \ldots-\sum_{i=1}^{c-1} \frac{i+1}{i^{3}}
$$

where $\zeta$ is the Riemann zeta function. Note that, given $\epsilon \in\left(0, \frac{1}{\alpha}\right)$, one may choose $c$ large enough so that $\delta>0$.

Proof of Theorem 9. Let $J:=\{2,3, \ldots,\lfloor\in n\rfloor\}$. Considering the visibility graph $G$ of $P$ and its subgraphs $G_{i}$ as defined previously, let $k$ be the minimum integer such that $\left|E\left(G_{k}\right)\right| \leqslant \alpha n$. If there is no such $k$ then let $k:=\lfloor\epsilon n\rfloor+1$. An integer $i \in J$ is large if $i \geqslant k$, and is small if $i \leqslant c$. An integer in $J$ that is neither large nor small is medium.

An $i$-pair is a pair of points in an $i$-line. A small pair is an $i$-pair for some small $i$. Define medium pairs and large pairs analogously, and let $P_{S}, P_{M}$ and $P_{L}$ denote the number of small, medium and large pairs respectively. An $i$-incidence is an incidence between a point of $P$ and an $i$-line. A small incidence is an $i$-incidence for some small $i$. Define medium incidences analogously, and let $I_{S}$ and $I_{M}$ denote the number of small and medium incidences respectively. Let $I$ denote the total number of incidences. Thus,

$$
I=\sum_{i \in J} i s_{i}
$$

The proof proceeds by establishing an upper bound on the number of small pairs in terms of the number of small incidences. Analogous bounds are proved for the number of medium pairs, and the number of large pairs. Combining these results gives the desired lower bound on the total number of incidences. 
For the bound on small pairs, Hirzebruch's Inequality is useful. Since no $\frac{n}{2}$ points are collinear and $n \geqslant 5$, there are no more than $n-3$ collinear points. Therefore, Hirzebruch's Inequality implies that $h s_{2}+\frac{3 h}{4} s_{3}-h n-h \sum_{i \geqslant 5}(2 i-9) s_{i} \geqslant 0$ since $h>0$. Thus,

$$
\begin{aligned}
P_{S} & =s_{2}+3 s_{3}+6 s_{4}+\sum_{i=5}^{c}\left(\begin{array}{l}
i \\
2
\end{array}\right) s_{i} \\
& \leqslant(h+1) s_{2}+\left(\frac{3 h}{4}+3\right) s_{3}+6 s_{4}+\sum_{i=5}^{c}\left(\begin{array}{l}
i \\
2
\end{array}\right) s_{i}-h n-h \sum_{i=5}^{c}(2 i-9) s_{i} \\
& \leqslant \frac{h+1}{2} \cdot 2 s_{2}+\frac{h+4}{4} \cdot 3 s_{3}+\frac{3}{2} \cdot 4 s_{4}+\sum_{i=5}^{c}\left(\frac{i-1}{2}-2 h+\frac{9 h}{i}\right) i s_{i}-h n .
\end{aligned}
$$

Setting $X:=\max \left\{\frac{h+1}{2}, \frac{h+4}{4}, \frac{3}{2}, \max _{5 \leqslant i \leqslant c}\left(\frac{i-1}{2}-2 h+\frac{9 h}{i}\right)\right\}$ implies that

$$
P_{S} \leqslant X I_{S}-h n
$$

The above inequality is strongest when $X$ is minimised by determining the optimal value of $h$ as follows. Let $\gamma(h, i):=\frac{i-1}{2}-2 h+\frac{9 h}{i}$. The second partial derivative of $\gamma(h, i)$ with respect to $i$ is positive for $i>0$, so $\gamma(h, i)$ is maximised for $i=5$ or $i=c$, and the other values of $i$ can be ignored. Thus $X$ is bounded from below by five linear functions of $h$. Notice that for fixed $c, \frac{h+1}{2}$ increases with $h$, while $\gamma(h, c)$ decreases with $h$. Therefore $X$ is at least the value of these functions at their intersection point, which occurs at $h=\frac{c(c-2)}{5 c-18}$. Note that this $h$ increases with $c$ for $c \geqslant 8$, so $h \geqslant \frac{24}{11}$. It is then easy to check that $X=\frac{h+1}{2}$ satisfies the other three constraints.

To bound the number of medium pairs, consider a medium $i \in J$. Since $i$ is not large, $\sum_{j \geqslant i}(j-1) s_{j}>\alpha n$. Hence, using parts (a) and (b) of the Szemerédi-Trotter Theorem,

$$
\sum_{j \geqslant i} j s_{j}=\sum_{j \geqslant i}(j-1) s_{j}+\sum_{j \geqslant i} s_{j} \leqslant \frac{\beta n^{2}}{2(i-1)^{2}}+\frac{\beta n^{2}}{2(i-1)^{3}}=\frac{\beta n^{2} i}{2(i-1)^{3}} .
$$

Given the factor $X$ in the bound on the number of small pairs in (1), it helps to introduce the same factor in the bound on the number of medium pairs. It will be convenient to define $Y:=c-1-2 X$.

$$
\begin{aligned}
P_{M}-X I_{M} & =\left(\sum_{i=c+1}^{k-1}\left(\begin{array}{l}
i \\
2
\end{array}\right) s_{i}\right)-X\left(\sum_{i=c+1}^{k-1} i s_{i}\right) \\
& =\frac{1}{2} \sum_{i=c+1}^{k-1}(i-1-2 X) i s_{i} \\
& =\frac{1}{2} \sum_{i=c+1}^{k-1}(i-c+Y) i s_{i} \\
& =\frac{1}{2}\left(\sum_{i=c+1}^{k-1} \sum_{j=i}^{k-1} j s_{j}\right)+\frac{Y}{2}\left(\sum_{i=c+1}^{k-1} i s_{i}\right) .
\end{aligned}
$$


Applying (2) yields

$$
P_{M}-X I_{M} \leqslant \frac{\beta n^{2}}{4}\left(Y \frac{c+1}{c^{3}}+\sum_{i \geqslant c} \frac{i+1}{i^{3}}\right) .
$$

It remains to bound the number of large pairs:

$$
P_{L}=\sum_{i=k}^{\lfloor\epsilon n\rfloor}\left(\begin{array}{l}
i \\
2
\end{array}\right) s_{i} \leqslant \frac{\epsilon n}{2} \sum_{i \geqslant k}(i-1) s_{i}=\frac{\epsilon n}{2}\left|E\left(G_{k}\right)\right| \leqslant \frac{\epsilon \alpha n^{2}}{2} .
$$

Combining (1), (3) and (4),

$$
\begin{aligned}
\left(\begin{array}{l}
n \\
2
\end{array}\right)=\frac{1}{2}\left(n^{2}-n\right) & \leqslant P_{S}+P_{M}+P_{L} \\
& \leqslant X I_{S}-h n+X I_{M}+\frac{\beta n^{2}}{4}\left(Y \frac{c+1}{c^{3}}+\sum_{i \geqslant c} \frac{i+1}{i^{3}}\right)+\frac{\epsilon \alpha n^{2}}{2} .
\end{aligned}
$$

Thus,

$$
I \geqslant I_{S}+I_{M} \geqslant \frac{1}{2 X}\left(1-\epsilon \alpha-\frac{\beta}{2}\left(Y \frac{c+1}{c^{3}}+\sum_{i \geqslant c} \frac{i+1}{i^{3}}\right)\right) n^{2}+\frac{2 h-1}{2 X} n .
$$

The result follows since $h \geqslant 1$.

It is worth noting that the methods used in the proof of Theorem 9 can be used to obtain good lower bounds on the number of edges in a visibility graph. The main difference is that edges are counted instead of incidences. For instance, we can prove the following result.

Theorem 10. Let $P$ be a set of $n$ points in the plane with at most $\frac{n}{50}$ collinear. Then the visibility graph of $P$ has at least $\frac{n^{2}}{50}$ edges.

For point sets with at most $o(n)$ collinear points, the following is the best asymptotic result we have obtained.

Theorem 11. Let $P$ be a set of $n$ points in the plane with at most $\ell$ collinear. Then the visibility graph of $P$ has at least $\frac{n^{2}}{39}-O(\ell n)$ edges. 


\section{A constant for Beck's Theorem}

Beck proved Theorem 5 as part of his work on Dirac's Conjecture [4]. Theorem 9 from the previous section and Lemmas 13 and 14 below can be used to give the best known constant in Beck's Theorem.

Theorem 12. Every set $P$ of $n$ points with at most $\ell$ collinear determines at least $\frac{1}{98} n(n-$

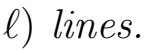

The following lemma, due to Kelly and Moser [11], is equivalent to Melchior's Inequality [14], which states that $s_{2} \geqslant 3+\sum_{i \geqslant 4}(i-3) s_{i}$. As before, $I$ is the total number of incidences in the arrangement of $P$. Let $E$ be the total number of edges in the visibility graph of $P$, and let $L$ be the total number of lines in the arrangement of $P$.

Lemma 13 (Kelly-Moser). If $P$ is not collinear, then $3 L \geqslant 3+I$, and since $I=E+L$, also $2 L \geqslant 3+E$.

When there is a large number of collinear points, the following lemma becomes stronger than Theorem 9 .

Lemma 14. Let $P$ be a set of $n$ points in the plane such that some line contains exactly $\ell$ points in $P$. Then the visibility graph of $P$ contains at least $\ell(n-\ell)$ edges.

Proof. Let $S$ be the set of $\ell$ collinear points in $P$. For each point $v \in S$ and for each point $w \in P \backslash S$, count the edge incident to $w$ in the direction of $v$. Since $S$ is collinear and $w$ is not in $S$, no edge is counted twice. Thus $E \geqslant|S| \cdot|P \backslash S|=\ell(n-\ell)$.

Proof of Theorem 12. Assume $\ell$ is the size of the largest collinear subset of $P$. If $\ell \geqslant \frac{n}{49}$ then $E \geqslant \frac{1}{49} n(n-\ell)$ by Lemma 14 and thus $L>\frac{1}{98} n(n-\ell)$ by Lemma 13. On the other hand, suppose $\ell \leqslant \frac{n}{49}$. Setting $c=67, \alpha=\frac{103}{16}, \beta=\frac{31827}{1024}$, and $\frac{\epsilon}{2}=\frac{\delta}{3}$ in Theorem 9 gives $\epsilon \geqslant \frac{1}{49}$ and $\delta \geqslant \frac{1}{32.57}$. So $I \geqslant \frac{1}{32.57} n^{2} \geqslant \frac{1}{32.57} n(n-\ell)$ and thus $L>\frac{1}{98} n(n-\ell)$ by Lemma 13.

A more direct approach similar to the methods used in the proof of Theorem 9 can be shown to improve Theorem 12 slightly to yield $\frac{1}{93} n(n-\ell)$ lines. The details are omitted, but can be found in [16].

Beck's Theorem is often stated as a bound on the number of lines with few points. In his original paper Beck [4] mentioned briefly in a footnote that Lemma 13 implies the following.

Observation 15 (Beck). If $P$ is not collinear, then at least half the lines determined by $P$ contain at most 3 points.

Proof. By Lemma 13,

$$
3 s_{2}+3 s_{3}+3 \sum_{i \geqslant 4} s_{i}>\sum_{i \geqslant 2} i s_{i} \geqslant 2 s_{2}+2 s_{3}+4 \sum_{i \geqslant 4} s_{i}
$$


Thus

$$
2\left(s_{2}+s_{3}\right)>\sum_{i \geqslant 2} s_{i}
$$

as desired.

Corollary 16. Every set $P$ of $n$ points with at most $\ell$ collinear determines at least $\frac{1}{196} n(n-\ell)$ lines each with at most 3 points.

\section{References}

[1] Martin Aigner and Günter M. Ziegler. Proofs from The Book. Springer, 3rd edn., 2004.

[2] Miklós Ajtai, Vašek Chvátal, Monroe M. Newborn, and Endre Szemerédi. Crossingfree subgraphs. In Theory and practice of combinatorics, vol. 60 of North-Holland Math. Stud., pp. 9-12. North-Holland, 1982.

[3] Jin Akiyama, Hiro Ito, Midori Kobayashi, and Gisaku Nakamura. Arrangements of $n$ points whose incident-line-numbers are at most n/2. Graphs Combin., 27(3):321-326, 2011. doi:10.1007/s00373-011-1023-4.

[4] József Beck. On the lattice property of the plane and some problems of Dirac, Motzkin and Erdős in combinatorial geometry. Combinatorica, 3(3-4):281-297, 1983. doi:10.1007/BF02579184.

[5] Wikipedia contributors. Beck's theorem (geometry). Wikipedia, the free encyclopedia, 2010. http://en.wikipedia.org/wiki/Beck's_theorem_(geometry).

[6] Gabriel A. Dirac. Collinearity properties of sets of points. Quart. J. Math., Oxford Ser. (2), 2:221-227, 1951. doi:10.1093/qmath/2.1.221.

[7] Paul Erdős. Some unsolved problems. Magyar Tud. Akad. Mat. Kutató Int. Közl., 6:221-254, 1961. http://www.renyi.hu/ p_erdos/1961-22.pdf.

[8] Paul Erdős and George Purdy. Some combinatorial problems in the plane. J. Combin. Theory Ser. A, 25(2):205-210, 1978. doi:10.1016/0097-3165(78)90085-7.

[9] Paul Erdős and George Purdy. Two combinatorial problems in the plane. Discrete Comput. Geom., 13(3-4):441-443, 1995. doi:10.1007/BF02574054.

[10] Friedrich Hirzebruch. Singularities of algebraic surfaces and characteristic numbers. In The Lefschetz Centennial Conference, Part I, vol. 58 of Contemp. Math., pp. 141-155. Amer. Math. Soc., 1986. doi:10.1090/conm/058.1/860410.

[11] Leroy M. Kelly and William O. J. Moser. On the number of ordinary lines determined by $n$ points. Canad. J. Math., 10:210-219, 1958. doi:10.4153/CJM-1958-024-6. 
[12] F. Thomson Leighton. Complexity Issues in VLSI. MIT Press, 1983.

[13] Ben D. Lund, George B. Purdy, and Justin W. Smith. A pseudoline counterexample to the Strong Dirac Conjecture. 2012. arXiv:1202.3110.

[14] Eberhard Melchior. Über Vielseite der projektiven Ebene. Deutsche Math., 5:461475, 1941.

[15] János Pach, Radoš Radoičić, Gábor Tardos, and Géza Tóth. Improving the crossing lemma by finding more crossings in sparse graphs. Discrete Comput. Geom., 36(4):527-552, 2006. doi:10.1007/s00454-006-1264-9.

[16] Michael S. Payne. Combinatorial geometry of point sets with collinearities. PhD thesis, The University of Melbourne, Department of Mathematics and Statistics, 2014. http://www.ms.unimelb. edu.au/ mspayne/MichaelPayneThesis.pdf.

[17] George Purdy. A proof of a consequence of Dirac's conjecture. Geom. Dedicata, 10(1-4):317-321, 1981. doi:10.1007/BF01447430.

[18] László A. Székely. Crossing numbers and hard Erdős problems in discrete geometry. Combin. Probab. Comput., 6(3):353-358, 1997. doi:10.1017/S0963548397002976.

[19] Endre Szemerédi and William T. Trotter, Jr. Extremal problems in discrete geometry. Combinatorica, 3(3-4):381-392, 1983. doi:10.1007/BF02579194. 